\title{
Relation between fractional order models and diffusion in the body
}

\author{
Dana Copot* Clara M. Ionescu* Robin De Keyser* \\ * Ghent University, Faculty of Engineering and Architecture, \\ Department of Electrical energy, Systems and Automation, \\ technologiepark 913, B9052 Zwijnaarde, Belgium (e-mail: \\ \{ClaraMihaela.Ionescu, Dana.Copot, Robain.DeKeyser\} @UGent.be).
}

\begin{abstract}
This paper presents the mathematical roots in fractional calculus that are present in the modeling of diffusion in the human body. Respiratory diffusion, as well as drug diffusion, are discussed and presented with their common mathematical framework. The links and discussion presented in this paper suggests that emerging tools from fractional calculus are natural solutions for modeling complex phenomena in biological tissue.
\end{abstract}

Keywords: fractional calculus, diffusion, modeling, respiratory system, fractional order impedance.

\section{INTRODUCTION}

Fractal time series analysis, and implicitly fractional calculus, have been used to improve the modeling accuracy of many phenomena in natural sciences (West (2013)). The fractional order derivative model is appropriate for modeling complex dynamics, eg. ions undergoing anomalous diffusion in dielectrics. Fractional order models are a generalizations of the classical integer order models. The net advantage os that fractional differ-integration enables the inclusion of memory and hereditary properties in models for processes governed by diffusion in all its aspects (i.e. sub-diffusion, classical and super-diffusion).

Non-integer order systems, also known as fractional order systems, have been introduced long ago in various fields of science for the modeling of diffusion processes such as electrochemical phenomena encountered in batteries (Lin et al. (2000); Sabatier et al. (2006)), the modeling of frequency effects (skin effect) in the squired-cages of induction machine and heat transfer. Such systems are characterized by long range memory transients (Gabano and Poinot (2009)). Fractional differential equation models are used to study anomalous diffusion; when a space fractional derivative is replaced by the second-order derivative in a diffusion model, it leads to super-diffusion (Meerschaert and Tadjeran (2009)).

There are many ongoing efforts in modeling diffusion by means of fractional calculus. Meerschaert et al. (Meerschaer et al. (2006)) gave a second-order accurate numerical approximation for the fractional diffusion equation, while Murio (Murio (2008)) discussed implicit finite difference approximation for time fractional diffusion equation. In the work of Lijuan et al. (Lijuan et al. (2009)), a finite difference-approximation for the fractional diffusion

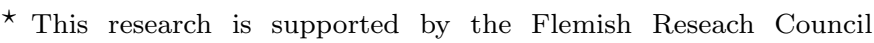
(FWO), Research Project FWOPR2013 005101. C.M. Ionescu is a post-doctoral fellow of FWO.
}

equation has been done using the Riemann-Liouville space fractional derivative.

Diffusion in the human body, such as respiratory or drug diffusion, is governed by power law semi-empirical models. Diffusion at cellular level is performed with energy loss, producing heat. In this paper, we employ heat unidirectional conduction in a homogeneous material with a spherical geometry. We first determine and analyze the nonpolynomial transfer functions characterizing the diffusion interface in the case of front face thermal characterization experiments which consist in measuring the temperature at the surface of a material where a random heat flux is applied.

Previous works concerning diffusion interface modeling using the fractional approach have already shown the ability of such models to estimate with good accuracy the frequency behavior from time data acquisition (Lin et al. (2000); Wang et al. (2002)). By analyzing the diffusion interface Bode plots in the case of the heat transfer through a finite thickness sphere, we outline the fractional behavior at high frequencies.

The paper is organized as follows: next section presents the mathematical framework for modeling diffusion and relation to fractional calculus. The third section presents biological principles of diffusion in the living tissue, i.e. respiratory diffusion and drug diffusion. The fourth section presents a discussion with respect to the use of these models in practice and a conclusion section summarizes the main outcome of this work.

\section{MATHEMATICAL FRAMEWORK}

Fractional calculus has been successfully employed in the last decennia to model biological systems and implicit complex phenomena, linking structure, morphology, geometry, with dynamical behavior and mechanical properties (West (2013); Magin (2006); Ionescu (2013); Ionescu et al. (2013)). 
We will start by introducing some basic functions. The gamma function is intrinsically tied to fractional calculus by definition. The simplest interpretation of the gamma function is the generalization of the factorial for all real numbers. The definition of the gamma function is given by:

$$
\Gamma(z)=\int_{0}^{\infty} e^{-u} u^{z-1} d u, \text { forall } z \in R
$$

The 'beauty' of the gamma function can be found in its properties:

$$
\begin{array}{r}
\Gamma(z+1)=z \Gamma(z) \\
\Gamma(z)=(z-1) !
\end{array}
$$

The consequence of this relation for integer values of $z$ is the definition for factorial. Using the gamma function we can also define the function $\Phi(\mathrm{t})$, which later will become useful for showing alternate forms of the fractional integral:

$$
\phi_{\alpha}(t)=\frac{t_{+}^{\alpha-1}}{\Gamma(\alpha)}
$$

Also known as the Euler Integral of the First Kind, the Beta Function is in important relationship in fractional calculus. Its solution not is only defined through the use of multiple Gamma Functions, but furthermore shares a form that is characteristically similar to the Fractional Integral/Derivative of many functions, particularly polynomials of the form $t^{\alpha}$ and the Mittag-Leffer Function:

$$
\begin{array}{r}
B(p, q)=\int_{0}^{1}(1-u)^{p-1} u q-1 d u=\frac{\Gamma(p) \Gamma(q)}{\Gamma(p+q)}=B(q, p) \\
\text { where } p, q \in R_{+}
\end{array}
$$

The Laplace Transform is a function transformation commonly used in the solution of complicated differential equations. With the Laplace transform it is frequently possible to avoid working with equations of different differential order directly by translating the problem into a domain where the solution presents itself algebraically. The formal definition of the Laplace transform is given as:

$$
\mathcal{L}\{f(t)\}=\int_{0}^{\infty} e^{-s t} f(t) d t=\tilde{f}(s)
$$

The Laplace Transform of the function $f(t)$ is said to exist if the above definition is a convergent integral. The requirement for this is that $f(t)$ does not grow at a rate higher than the rate at which the exponential term $e^{-s t}$ decreases. One important property of the Laplace transform that should be addressed is the Laplace transform of a derivative of integer order $n$ of the function $f(t)$, given by:

$$
\begin{aligned}
\mathcal{L}\left\{f^{n}(t)\right\} & =s^{n} \tilde{f}(s)-\sum_{k=0}^{n-1} s^{n-k-1} f^{(0)}= \\
& =s^{n} \tilde{f}(s)-\sum_{k=0}^{n-1} s^{k} f^{(n-k-1)}(0)
\end{aligned}
$$

The Mittag-Leffer function is an important function that finds widespread use in the world of fractional calculus (Podlubny (1999)). Just as the exponential naturally arises out of the solution to integer order differential equations, the Mittag-Leffer function plays an analogous role in the solution of non-integer order differential equations. In fact, the exponential function itself is a very specific form, one of an infinite set, of this seemingly ubiquitous function. The standard definition of the Mittag-Leffer is:

$$
E_{\alpha}(z)=\sum_{k=0}^{\infty} \frac{z^{k}}{\Gamma(\alpha k+1)}, \alpha>0
$$

The fractional time derivative is given by Podlubny (1999):

$$
D_{t}^{\alpha}[f(t)]=\frac{1}{\Gamma(n-\alpha)} \frac{d^{n}}{d t^{n}} \int_{0}^{\tau} \frac{f\left(t^{\prime}\right) d t^{\prime}}{\left(t-t^{\prime}\right)^{(1-\alpha-n)}}
$$

where $[\alpha]+1 \geq n \geq[\alpha]$ and the bracket denotes the closest integer value to $\alpha$. Relation (8) denotes the RiemannLiouville fractional differential operator.

Generalization of the relaxation function to its fractional order form is given by (West (2013)):

$$
D_{t}^{\alpha}[Y(t)]+\lambda^{\alpha} Y(t)=\frac{t^{-\alpha} Y(0)}{\Gamma(1-\alpha)}
$$

where the dissipation term $\lambda^{\alpha}$ is positive definite. Analytical solutions for this kind of equations have been developed by several research groups, notably in (Miller and Ross (1993)). Taking the Laplace transform of (9) one obtains:

$$
\hat{Y}(s)=\frac{Y(0)}{s} \cdot \frac{s^{\alpha}}{s^{\alpha}+\lambda^{\alpha}}
$$

Re-writing relation (9) in terms of the Mittag-Leffler definition from $(7)$ we have that:

$$
Y(t)=Y(0) E_{\alpha}\left(-(\lambda t)^{\alpha}\right)=Y(0) \sum_{k=0}^{\infty} \frac{-1^{k}}{\Gamma(\alpha k+1)}(\lambda t)^{k \alpha}
$$

which for $\alpha=1$ results in the exponential function:

$$
\lim _{\alpha \rightarrow 1} E_{\alpha}\left(-(\lambda t)^{\alpha}\right)=e^{-\lambda t}
$$

which is indeed the case of (9) when $\alpha \rightarrow 1$. The MittagLeffler function from (7) can be used to express long-time memory associated with fractional relazation process:

$$
\lim _{t \rightarrow \infty} E_{\alpha}\left(-(\lambda t)^{\alpha}\right)=(\lambda t)^{-\alpha}
$$

which denotes an inverse power law. 
It has been shown that diffusion process obey power law distribution (Magin (2006)).

\section{DIFFUSION PRINCIPLES}

In this section we shall introduce the biological framework of diffusion occurring in the human body. Two examples are given: i) diffusion in the respiratory system for gas exchange and ii) drug diffusion during anesthesia.

\subsection{Respiratory diffusion}

This complex phenomena is part of an intricate system of airways and lung parenchyma which has the purpose to facilitate air flow into the body to the capillaries. It has been shown that a structural, geometrical and morphological modeling leads to impedance models of fractional order (Ionescu (2013)). In this paper, the basic bio-chemical processes are lined-out for motivating diffusion to be modeled by fractional order derivatives.

Carbon dioxide $\left(\mathrm{CO}_{2}\right)$ transport: The systemic capillaries deliver oxygen to the tissues and remove carbon dioxide. About $8 \%$ of the $\mathrm{CO}_{2}$ in blood is simply dissolved in plasma; another $20 \%$ is bound to hemoglobin. The remaining $72 \%$ of the $\mathrm{CO}_{2}$ diffuses into the red blood cells, where the enzyme carbonic anhydrase catalyzes the combination of $\mathrm{CO}_{2}$ with water to form carbonic acid $\left(\mathrm{H}_{2} \mathrm{CO}_{3}\right)$. Carbonic acid dissociates into bicarbonate $\left(\mathrm{HCO}_{3}\right)$ and hydrogen $\left(H^{+}\right)$ions. The $H^{+}$binds to deoxyhemoglobin, and the bicarbonate moves out of the erythrocyte into the plasma via a transporter that exchanges one chloride ion for a bicarbonate (this is called the chloride shift). This reaction removes large amounts of $\mathrm{CO}_{2}$ from the plasma, facilitating the diffusion of additional $\mathrm{CO}_{2}$ into the plasma from the surrounding tissues. The formation of carbonic acid is also important in maintaining the acid-base balance of the blood, because bicarbonate serves as the major buffer of the blood plasma (Levitzky (2007)). The blood carries $\mathrm{CO}_{2}$ in these forms to the lungs. The lower $\mathrm{PCO}_{2}$ of the air inside the alveoli causes the carbonic anhydrase reaction to proceed in the reverse direction, converting $\mathrm{H}_{2} \mathrm{CO}_{3}$ into $\mathrm{H}_{2} \mathrm{O}$ and $\mathrm{CO}_{2}$. The $\mathrm{CO}_{2}$ diffuses out of the red blood cells and into the alveoli, so that it can leave the body in the next exhalation.

Oxygen transport: The $P_{O 2}$ of the air within alveoli at sea level is approximately 105 millimeters of mercury ( $\mathrm{mm}$ $\mathrm{Hg}$ ), which is less than the $P_{\mathrm{O} 2}$ of the atmosphere because of the mixing of freshly inspired air with old air in the anatomical dead space of the respiratory system. The $P_{\mathrm{O} 2}$ of the blood leaving the alveoli is slightly less than this, about $100 \mathrm{~mm} \mathrm{Hg}$, because the blood plasma is not completely saturated with oxygen due to slight inefficiencies in lung function. At a blood $P_{O 2}$ of $100 \mathrm{~mm} \mathrm{Hg}$, approximately $97 \%$ of the hemoglobin within red blood cells is in the form of oxyhemoglobinindicated as a percent oxyhemoglobin saturation of $97 \%$. As the blood travels through the systemic blood capillaries, oxygen leaves the blood and diffuses into the tissues. Consequently, the blood that leaves the tissue in the veins has a $P_{O 2}$ that is decreased (in a resting person) to about $40 \mathrm{~mm} \mathrm{Hg}$. At this lower $P_{O 2}$, the percent saturation of hemoglobin is only $75 \%$ (Levitzky (2007)). In a person at rest, therefore,
$22 \%$ of the oxyhemoglobin has released its oxygen to the tissues. Put another way, roughly one-fifth of the oxygen is unloaded in the tissues, leaving four-fifths of the oxygen in the blood as a reserve. In figure 1a the gas exchange in the blood capillaries of the lungs and systemic circulation is shown. As a result of gas exchange in the lungs, the systemic arteries carry oxygenated blood with a relatively low carbon dioxide concentration. After the oxygen is unloaded to the tissues, the blood in the systemic veins has a lowered oxygen content and an increased carbon dioxide concentration. In figure $1 b$ the heat transfer trough a sphere is represented (Levitzky (2007)).
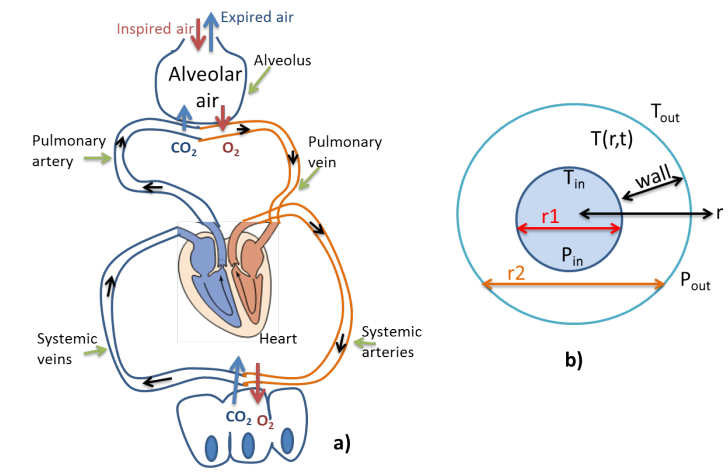

b)

Fig. 1. a) Gas exchange in the blood capillaries of the lungs and systemic circulation and b) heat transfer trough a sphere.

Sphere heat transfer (diffusion) modeling for the respiratory system: Let us consider the problem which consists in computing the heating temperature $T_{i n}(t)$ at the inner surface of a sphere of radius $r_{1}$ where a random heat flux $\phi$ in is applied. Heating temperature $T(r, t)$ is assumed to be uniform on any spherical slice of radius $r$ (see figure 1b). Let $\phi(r, t)$ to be the flux passing through the sphere at radius $r$. Assuming the heat conduction is isotropic, the heat diffusion is an unidirectional phenomenon governed by the following equations, which can be written, using spherical coordinates:

$$
\begin{gathered}
\frac{\partial T(r, t)}{\partial t}=a\left[\frac{\partial^{2} T(r, t)}{\partial r^{2}}+\frac{2}{r} x \frac{\partial T(r, t)}{\partial r}\right] \\
\Phi(r, t)=\lambda\left(4 \pi r^{2}\right) \frac{\partial T(r, t)}{\partial r}
\end{gathered}
$$

where:

$\lambda$ : thermal conductivity $\left(W m^{-1} C^{-1}\right)$, $a=\lambda / \rho c$ : thermal diffusivity $\left(m^{2} s^{-1}\right)$, $\rho$ : mass density $\left(\mathrm{kgm}^{-3}\right)$, $c$ : specific heat $\left(J \mathrm{~kg}^{-1} \mathrm{C}^{-1}\right)$.

Let $T_{\text {out }}(t)$ to be the outer surface temperature and $\phi_{\text {out }}(t)$ the output flux. We write $T_{i n}(s), \phi_{i n}(s)$ and $T_{\text {out }}(s)$, $\phi_{\text {out }}(s)$ the Laplace transforms of $T_{i n}(t), \phi_{\text {in }}(t)$ at $r=r_{1}$ and $T_{\text {out }}(t), \phi_{\text {out }}(t)$ at $r=r_{2}$. The wall modeling can be done using a thermal quadrupole relating the inputs $T_{\text {in }}(s), \phi_{\text {in }}(s)$ to the outputs $T_{\text {out }}(s), \phi_{\text {out }}(s)$ (Wang et al. (2002); Maillet et al. (2000)).

As in the case of the alveolar wall, we can establish the terms A, B, C, D of the matrix characterizing the heat transfer of the sphere. 


$$
\begin{array}{r}
A=\frac{r_{1}}{r_{2}} \cosh \left(\delta\left(r_{2}-r_{1}\right)\right)-\frac{\sinh \left(\delta\left(r_{2}-r_{1}\right)\right)}{\delta r_{1}} \\
B=\frac{1}{4 \pi r_{1} r_{2}} \frac{\sinh \left(\delta\left(r_{2}-r_{1}\right)\right)}{\delta \lambda} \\
C=4 \pi r_{2} \lambda\left[1-\frac{r_{1}}{r_{2}} \cosh \left(\delta\left(r_{2}-r_{1}\right)\right)+\right. \\
+\left(\lambda r_{1}-\frac{1}{\lambda r_{2}}\right) \frac{\sinh \left(\delta\left(r_{2}-r_{1}\right)\right)}{\delta r_{1}} \\
D=\frac{r_{1}}{r_{2}} \cosh \left(\delta\left(r_{2}-r_{1}\right)\right)+\frac{\sinh \left(\delta\left(r_{2}-r_{1}\right)\right)}{\delta r_{1}}
\end{array}
$$

At high frequencies, the thermal impedance of the sphere behaves like a non-integer integrator whose order is equal to 0.5 :

$$
\lim _{s \rightarrow \infty} Z_{s}(s)=\frac{\sqrt{a}}{\lambda\left(4 \pi r_{i}^{2}\right) s^{0.5}}
$$

which motivates us to consider that indeed, respiratory diffusion with loss of energy and heat production, can be modeled by fractional order impedance models.

\subsection{Drug diffusion}

From the point-of-view of regulating anesthesia, mathematical modeling of drug diffusion can be very useful to better understand the mechanism of drug delivery systems. Therefore, the description of drug transport into the human body can be highly beneficial (Siepman and Siepmann (2008); Siepmann and Goepferich (2001); Baker (1987)) from two points-of-view: (i) allows you to understand the insight of the mechanism and (ii) allows you to do quantitative prediction of the effects of formulation and processing parameters on the resulting drug release kinetics. The major challenge in the development or optimization of automated drug delivery systems is to achieve optimal drug concentration at the site of action. In order to have the optimal concentration-time-profile at the site of action the release of the drug must be controlled as accurately as possible.

Diffusional behavior is based on the assumption that the molecules perform a random walk in the space that is available to them, this is called Brownian motion (Berg (1993)). The nature of this random walk is such that the molecules have equal chance to go either way (in the absence of external fields). In its classical form, diffusion is described by Fick's law and strong experimental evidence of diffusion processes which deviate from this law has been found (Resigno (2004)). Diffusion process represents a very important role in many areas of research, such as: chemistry, biology an physics and the results of this process is to mix and move the molecules from one point to another, which have different concentration gradients.

Compartmental models for pharmacokinetics (PK) have been generalized using fractional calculus in order to extend the systems to the form of fractional-order differential equations (Dokoumetzidis and Macheras (2009); Popovic et al. (2010)). PK can be defined as the study of drug disposition in the body and it focuses on drug plasma (blood) amount changes. The plasma amount of any type of drug depends on three processes: absorption, distribution and elimination. These processes will make the amount rise and fall according to their rates. Absorption is related to the movement of the drug into the bloodstream. Its rate depends on the physical characteristics of the drug and the drug's chemical formula. Distribution is defined as the process where a drug leaves the bloodstream and goes into neighboring organs and tissues (Higuchi (1961)).

Considering a two-compartmental model depicted in figure 2. Assuming that $q_{i}(t)=v_{i} c_{i}$, for $i=1,2$, denote the amount of a drug in a specific compartment. Therefore, $c_{i}$ is the concentration of a drug, given by $c_{i}=q_{i} / v_{i}$ and $v_{i}$ is the volume of compartment $i . K_{i j}$ represent the given constants of elimination from compartment $i$ to compartment $j$. The first compartment represents the place where the drug is applied (plasma) and the second compartment represents the target organ (muscle). The basic idea behind this model can be used to model the diffusion process in the human body by a multi-compartmental model (Dokoumetzidis and Macheras (2009); Popovic et al. (2010); Copot et al. (2013)). The model is formulated while maintaining the mass balance principles.

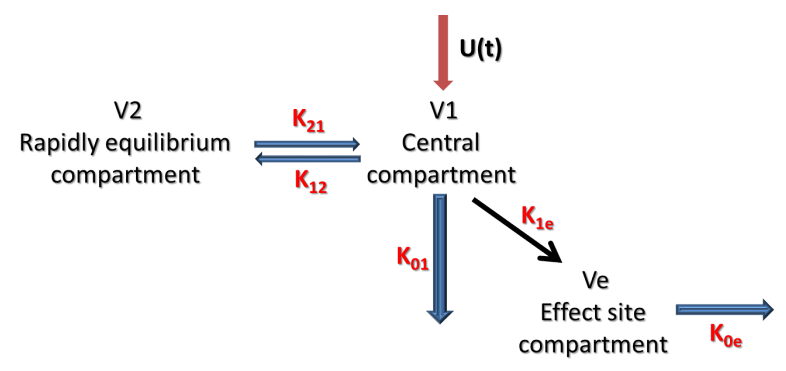

Fig. 2. A two compartmental model representation.

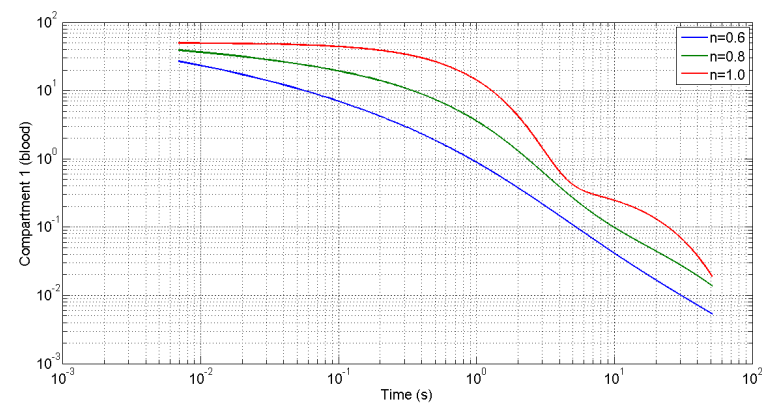

Fig. 3. Drug diffusion as a function of time for compartment 1 (i.e. blood).

Figure 3 shows the results of anesthetic drug diffusion in the human body in compartment 1 (i.e. blood). In this figure, we can observe that various dynamics can be captured simply by modifying the value of the fractional order $n$. The red line represent the result for a value of $n=1$, green line a value of $n=0.8$ and the blue line a value of $n=0.6$. Notice that both $\mathrm{X}$ and $\mathrm{Y}$ scales are logarithmic. This is of particular interest when capturing inter-patient variability and dynamics between different mass compartments (i.e. blood, muscle, fat) which have significantly different dynamics. 
Effect to drug concentrations curves are very important to characterize the sensitivity of the patient to the administered drug. The relation between the effect site concentration $V_{e}$ from figure 2 and its effect is given by a nonlinear sigmoid Hill curve:

$$
\operatorname{Effect}(t)=E_{0}-E_{\max } \cdot \frac{V_{e}^{\gamma}(t)}{V_{e}^{\gamma}(t)+V_{5} 0^{\gamma}}
$$

where $E_{0}$ is the effect value when the patient is awake; $E_{\max }$ is the maximum effect that can be achieved by the infusion of anesthetic drug; $V_{50}$ is the anesthetic drug concentration at half of the maximum effect and $\gamma$ is a parameter which together with the $V_{50}$ determines the patient sensitivity to the drug. $E_{0}$ and $E_{\max }$ are usually considered equal to 100 .

Based on (13) one can write the same relationship for the Hill curve:

$$
\frac{E f f e c t(t)}{V_{e}(t)}=k \cdot t^{n}
$$

where $k$ and $n$ are varying on the patient PK-PD characteristics. If one compares (21) with (22) it can be recognized the resemblance in the power term and observe in fact a simplification of the model from (21) in terms of parameter number. From a structural point of view, there is no difference between the models, since both are semiempirical models. The term $\frac{E f f e c t(t)}{V_{e}(t)}$ denotes the effect-toconcentration ratio (ECR) and its units are $[\% / \mathrm{mg} / \mathrm{ml}]$. Based on the principles of fractal walk, if the scale of the ECR representation is changed from linear to logarithmic, it becomes a (quasi)linear relation.

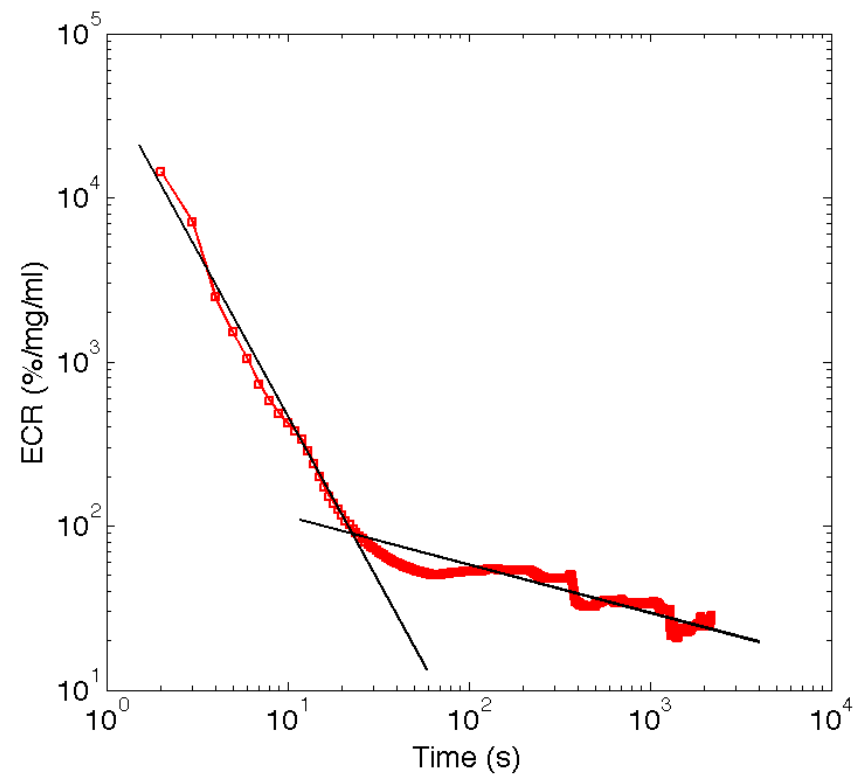

Fig. 4. Example of ECR curve for arbitrary patient model parameter values.

Notice that in figure 4 both $\mathrm{X}$ and $\mathrm{Y}$ scales are logarithmic. Observe that the ECR is indeed quasi-linear and difference in slope can be used to account for the variations in the dynamic profiles of $V_{e}(t)$ which depend on patient's drug intake time-varying dynamics.

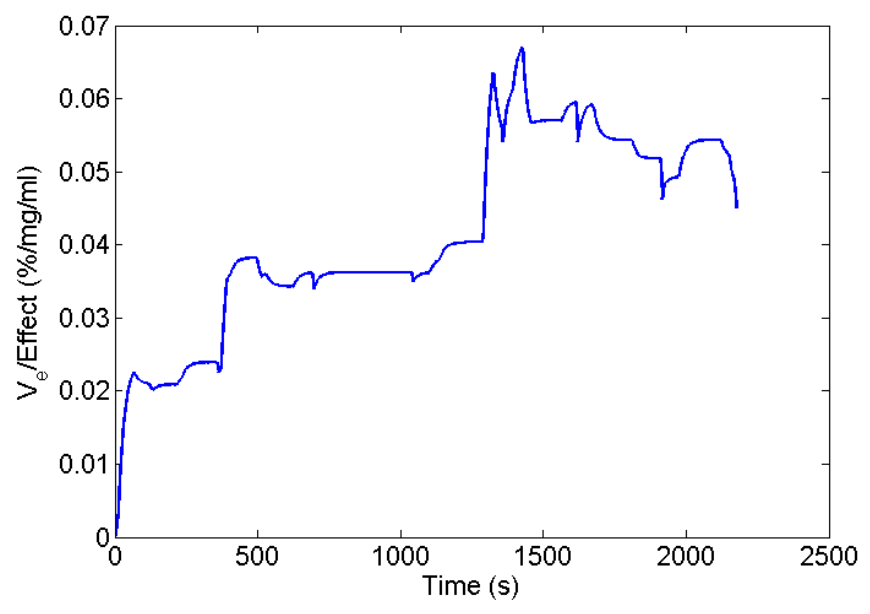

Fig. 5. Example of the response $\left(V_{e} /\right.$ Effect) curve.

In figure 5 the response $\left(V_{e}(t) / E f f e c t(t)\right)$ is shown, i.e. the inverse of (22). This dynamic behavior can be easily approximated by transfer functions with gain-pole-zero structure. This approach has also been contemplated in (Heusden et al. (2014)).

\section{CHALLENGES}

A first problem is to find online adaptation algorithms which may adapt the model parameters $k$ and $n$ to the patient characteristics. Identification from logarithmically sampled data has been proposed in (De Keyser et al. (2011)) and represents a good framework for developing the online identification algorithm.

The next problem is to find a solution to integrate the model from (22) into a closed loop control system taking into account the requirement for a logarithmic sample time (i.e. in order to maintain linearity). Although it may look surprisingly, it has already been shown in various examples that a Riemann sampling rate (i.e. linear periodic) may be outperformed by a Lebesque sampling rate (i.e. eventtriggered) in several applications (Goodwin et al. (2013)). Since the Lebesque sampling rate is an event triggered rate used successfully in practice in closed loop control (e.g. networked control, sensor networks), it may be revealing to look into the possibility of a logarithmic sampling rate.

\section{CONCLUSIONS}

This paper presented the available tools emerging from fractional calculus to model the nonlinear characteristics of the diffusion phenomena in the human body. Two example shave been given and related to principles of fractional calculus: i) respiratory diffusion and ii) drug diffusion. Advantages and challenges have been discussed.

Results suggest that the high degree of inter-patient variability and nonlinearity may be avoided, leading to linear control techniques instead of advanced, complex control techniques. Further steps of this research line are dealing with the current limitations: i) online identification of logarithmically sampled data and ii) control of logarithmically sampled systems. 


\section{REFERENCES}

Baker, R. (1987). Controlled Release of Biologically Active Agents,. John Wiley \& Sons, New York.

Berg, H. (1993). Random Walk in Biology,. Princenton University Press, Princenton NJ.

Copot, D., Chevalier, A., Ionescu, C., and De Keyser, R. (2013). A two-compartment fractional derivative model for propofol diffusion in anesthesia. Conference on Systems and Control, Hyderabad, India, 28-31 August, 264-269.

De Keyser, R., Ionescu, C., and Festila, C. (2011). A onestep procedure for frequency response estimation based on switch-mode transfer function analyzer. Proceedings IEEE-CDC Conference on Decision and Control joint with ECC-European Control Conference, December 1215, Orlando, Florida, 1189-1194.

Dokoumetzidis, A. and Macheras, P. (2009). Fractional kinetics in drug absorption and disposition processes. Journal of Pharmacokinetics and Pharmacodynamics, $36,165-178$.

Gabano, J.D. and Poinot, T. (2009). Fractional identification algorithms applied to thermal parameter estimation,. 15th IFAC Symposium on System Identification.

Goodwin, G., Aguero, J., Cea-Garrido, M., Salgado, M., and Yuz, J. (2013). Sampling and sampled-data models. IEEE Control Systems Magazine, 33, 34-53.

Heusden, K., Dumont, G., Soltesz, K., Petersen, C., Umedaly, A., West, N., and Ansermino, J. (2014). Design and clinical evaluation of robust pid control of propofol anesthesia in children. IEEE Transactions on Control Systems Technology, 22, 491-501.

Higuchi, T. (1961). Rate of release of medicaments from ointment bases containing drugs in suspension. Journal of Pharmaceutical Sciences, 50, 874-875.

Ionescu, C. (2013). The human respiratory system: an analysis of the interplay between anatomy, structure, breathing and fractal dynamics. Springer, Series in BioEngineering.

Ionescu, C., Copot, D., and De Keyser, R. (2013). Three compartmental model for propofol diffusion during general anesthesia. Journal of Discontinuity, Nonlinearity and Complexity, 2, 357-368.

Levitzky, M. (2007). Pulmonary Physiology. McGraw-Hill Companies Inc., New York.

Lijuan, S., Wenqia, W., and Zhoxima, Y. (2009). Finite difference approximations for the fractional advectiondiffusion equation,. Physics Letters A, 373, 4405-4408.

Lin, J., Poinot, T., Trigeassou, J.C., and Ouvrard, R. (2000). Parameter estimation of fractional systems: application to the modeling of a lead-acid battery,. 12th IFAC Symposium on System Identification.

Magin, R. (2006). Fractional Calculus in Bioengineering. Begell House Publishers.

Maillet, D., Andre, S., Batsale, J.C., Degiovanni, A., and Moyne, C. (2000). Thermal Quadrupoles: Solving the Heat Equation through Integral Transforms. Wiley, New York.

Meerschaer, M., Scheffler, H., and Tadjeran, C. (2006). Finite difference methods for two-dimensional fractional dispersion equation,. Journal of Computational Physics, 211, 249-261.

Meerschaert, M. and Tadjeran, C. (2009). Finite difference approximations for fractional advection-dispersion flow equations,. Journal of Computational and Applied Mathematics, 172, 65-77.

Miller, K. and Ross, B. (1993). An introduction to fractional calculus and fractional differential equations. John Wiley and Sons, New York.

Murio, D. (2008). Implicit finite difference approximation for time fractional diffusion equations,. Computers and Mathematics with Applications, 56, 1138-1145.

Podlubny, I. (1999). Fractional differential equations. Academic Press, San Diego.

Popovic, K., Atanackovic, M.T., Pilipovic, A.S., Rapaic, M.R., Pilipovic, S., and Atanackovic, T.M. (2010). A new approach to the compartmental analysis in pharmacokinetics: fractional time evolution of diclofenac. Journal of Pharmacokinetics and Pharmacodynamics, $37,119-134$.

Resigno, A. (2004). Foundations of pharmacokinetics,. Kluwer, New York.

Sabatier, J., Aoun, M., Oustaloup, A., Gregoire, G., Ragot, F., and Roy, P. (2006). Fractional system identification for lead acid battery state charge estimation,. Signal Processing, 10, 2645-2657.

Siepman, J. and Siepmann, F. (2008). Mathematical modeling of drug delivery,. International Journal of Pharmaceutics, 364, 328-343.

Siepmann, J. and Goepferich, A. (2001). Mathematical modeling of bioerodible, polymeric drug delivery systems,. Advanced Drug Delivery Review, 48, 229-247.

Wang, H., Degiovanni, A., and Moyne, C. (2002). Periodic thermal contact: a quadrupole model and an experiment,. International Journal of Thermal Sciences, 41, $125-135$.

West, B. (2013). Fractal physiology and the fractional calculus: a perspective. Frontiers in Physiology. 